\title{
LETTERS
}

\section{Letter to the editor: Commitment needed for the prevention of congenital rubella syndrome in Europe}

T Derrough (tarik.derrough@ecdc.europa.eu) ${ }^{1}$, S Bacci $^{1}$, P L Lopalco ${ }^{1}$

1. European Centre for Disease Prevention and Control (ECDC), Stockholm, Sweden

The ongoing outbreak of rubella in Romania as reported by Janta et al. [1] highlights the public health efforts that are yet necessary to meet the World Health Organization (WHO) Regional Office for Europe target for the elimination of measles and rubella and prevention of congenital rubella syndrome (CRS) by 2015 [2].

Ongoing measles outbreaks being reported in the European Union/European Economic Area (EU/EEA) countries is a reminder that rubella outbreaks may be ongoing concomitantly despite apparent lower number of cases reported through routine surveillance in the EU [3].

Rubella vaccines have been used in the EU/EEA countries for more than 20 years. Currently all EU/EEA countries have adopted a combined measles-mumpsrubella (MMR) vaccination with two doses in the childhood vaccination schedule.

At initial implementation, rubella vaccination followed two general approaches: either a targeted immunisation of adolescent girls and/or women of childbearing age, with the aim of reducing the incidence of CRS, or a more global approach that targeted young infants, susceptible adolescents and adults in order to interrupt rubella transmission and eliminate rubella as well as CRS. The first approach requires that virtually every susceptible woman should be immunised to reach elimination of CRS. The latter approach is the current standard for MMR vaccination in EU/EEA countries. Currently available MMR vaccines are not expensive, highly efficacious and well tolerated in all age groups [4].

To ensure the benefits of universal vaccination with two doses of MMR, a strong long-term public health and political commitment is vital. This is required for achieving and maintaining high vaccination coverage, for ensuring continuous vaccine supply and delivery and also for having adequate surveillance activities to monitor trends in CRS and rubella and the effects of interventions.
Screening for rubella antibodies - as part of pre-conceptual or antenatal care to identify unprotected women - enables for active rubella vaccination offer in order to protect future pregnancies. This might represent an additional tool for CRS prevention [5]. However, high vaccination coverage in childhood and reliable immunisation records should be the primary aim to strive for.

In the past, outbreaks in young adults in EU/EEA countries led to children being born with CRS [6]. This situation is likely to repeat if women of childbearing age are infected with rubella. All possible efforts should be undertaken to prevent any case of CRS in Europe in the future. Commitment is now needed at political and public health level in order to ensure that the potentially debilitating consequences of rubella infection in pregnancy for children born in the EU represent a story of the past.

The epidemiology and profile of population susceptibility for both measles and rubella are different and require different catch-up strategies to reach the elimination goal. In many countries, rubella vaccination was introduced later than measles, with different segments of the population being initially targeted by vaccination - therefore leaving cohorts of individuals unprotected. By contrast, measles vaccination has always been targeting toddlers, young children and adolescents. The initiatives undertaken in delivering measles vaccination programmes and the use of combined vaccines may provide an opportunity for synergy in activities towards rubella and CRS elimination. However, rubella deserves its own attention and public health initiatives, as susceptible population groups might be different than those unprotected towards measles.

The European Centre for Disease Prevention and Control (ECDC) is committed to support the EU/EEA Member States in the measles, rubella and CRS elimination goal. In the coming months and years, ECDC will work closely with the Member States, the European Commission and the WHO Regional Office for Europe to help ensure that appropriate measures are implemented and adequate 
virological and epidemiological surveillance mechanisms are in place for rubella, as well as for measles.

\section{References}

1. Janta D, Stanescu A, Lupulescu E, Molnar G, Pistol A.

Ongoing rubella outbreak among adolescents in Salaj, Romania, September 2011-January 2012. Euro Surveill. 2012;17(7): pii=20089. Available from: http://www. eurosurveillance.org/ViewArticle.aspx?Articleld $=20089$

2. World Health Organization Regional Office for Europe (WHO). Renewed commitment to measles and rubella elimination and prevention of congenital rubella syndrome in the WHO European Region by 2015. Copenhagen: WHO. 23 Jul 2010. Available from: http://www.euro.who.int/_data/assets/pdf_ file/0008/119546/RC60_edoc15.pdf

3. European Centre for Disease Prevention and Control (ECDC). EUVAC-Net. Surveillance. Stockholm: ECDC. [Accessed 8 Mar 2012]. Available from: http://ecdc.europa.eu/en/activities/ surveillance/euvac/data/Pages/reports.aspx

4. World Health Organization (WHO). Rubella vaccines: WHO position paper. Wkly Epidemiol Rec. 2011;86(29):301-16.

5. World Health Organization (WHO). What is the effectiveness of antenatal care? (Supplement). Copenhagen: WHO. Dec 2005. Available from: http://www.euro.who.int/__data/assets/pdf_ file/0005/7466o/E87997.pdf

6. Plotkin SA, Reef SE. Rubella vaccine. In: Plotkin SA, Orenstein WA, Offit P, editors. Vaccines 5th ed. Saunders: Elsevier. 2008. p. 735-71. 